\section{俩 Heighten Science \\ P U B L I C I T I I O N S Corporation ISSN \\ 2639-6629}

\title{
What is the Cost of Measuring a Blood Pressure?
}

\author{
Steven A Yarows* \\ Chelsea Family and Internal Medicine, Adjunct Professor of Internal Medicine, Michigan Medicine, \\ IHA, 128 Van Buren, Chelsea, MI 48118, USA
}

\begin{abstract}
*Address for Correspondence: Steven A Yarows, MD, FACP, FASH, FAHA, Chelsea Family and Internal Medicine, Adjunct Professor of Internal Medicine, Michigan Medicine, IHA, 128 Van Buren, Chelsea, MI 48118, USA, Tel: (734) 475-8677;

Email: steven_yarows@ihacares.com

Submitted: 27 September 2018

Approved: 10 October 2018

Published: 11 October 2018
\end{abstract}

Copyright: (๑) 2018 Yarows SA. This is an open access article distributed under the Creative Commons Attribution License, which permits unrestricted use, distribution, and reproduction in any medium, provided the original work is properly cited.

Keywords: Blood pressure; Methods/ determination; Physicians' offices; Primary health care; Automation; Cost of blood pressure; Efficiency/cost reductio; Medical devices

Abbreviations: BP: Blood Pressure; MA: Medical Assistant; BMI: Body Mass Index; WA: Welch Allyn Connex Vital Signs Monitor/Oscillometric Method; PCP: Primary Care Practice

Check for updates

\section{Abstract}

Rationale: Blood Pressure measurement has transitioned to the oscillometric method in most hospitals in the United States, however out-patient offices mainly use the auscultatory technique.

Objective: To determine time taken to measure blood pressure by an automatic oscillometric device compared to an auscultatory measurement device and to determine what each measurement costs.

Methods: Blood Pressures were measured in a single primary care office by medical assistants (MA) for patients seen for office visits. Timed measurements were performed using an automated oscillometric Welch Allyn Connex Vital Signs Monitor (WA) and manually using a Tycos device. A minimum of 400 readings were taken with each method.

Results: The average time to manually measure BP was 58.6 seconds, whereas the WA average was 39.8 seconds, 18.8 seconds faster $(p<0.05)$. There was an improvement in measurement time with MA experience with the WA device $(\mathrm{p}<0.05)$. The average MA cost to measure a single BP using the manual method was $\$ 0.35$ vs. the WA method ( $\$ 0.24)$ or a savings of $\$ 0.11$ per measurement. The improvement with experience of WA method reduced cost to $\$ 0.17$ per measurement.

Conclusion: The oscillometric method saved 17 cents per measurement potentially saving $\$ 1,119$ per year for our primary care practice.

\section{Introduction}

The measurement of vital signs is the standard of care for every patient in Primary care physicians (PCP) offices. Vital signs include weight and blood pressure (BP) for each patient and height, pulse, finger oximetry, and temperature intermittently, as indicated. In 2015, there were an estimated 922.6 million physician office visits with PCP providing 331.2 million encounters or 53\% of all visits [1]. The most common reason was hypertension.

In the mid-1990's most hospital vital signs were silently transitioned from manual to automatic electronic measurement likely due to efficiency without expert guidelines nor clinical studies indicating this preference. Office measurements have never been standardized and are more slowly transitioning to the electronic method likely due to cost. Canada has been actively funding and promoting automatic electronic blood pressure monitoring without an observer present [2]. The positive results of the largest BP outcome trial of 9,361 utilizing solely an electronic automatic device may increase the purchase of these type of devices in Primary Care offices to more effectively control hypertension [3].

PCP provide high-volume care with high overhead making efficiency critical. The decision to purchase expensive equipment must be mitigated by the return on the investment. The type of PCP office (private-practice, academic, teaching, Hospital 
owned, Government owed, etc.) and availability of capital purchase funds also determine the readiness of purchases. Staff salaries and benefits are the major cost in PCP practices and examination room availability are often limited. Automatic office BP machines are relatively expensive ranging from $\$ 650$ to over $\$ 2,000$ per unit and the number of units required per practice is determined by the number of medical assistants per office.

Despite the transition of measurements indicated above, there is no literature reporting the actual time nor the costs by either the oscillometric or auscultatory method of measuring a BP in a clinical office. This analysis could determine if a capital investment of an automatic oscillometric BP monitor is more cost-effective rather than the standard auscultatory measurement techniques. This study was designed to determine the cost of measuring BP in a clinical practice for the determination of the return on investment of the oscillometric method.

\section{Methods}

\section{Study design}

This study was a quality-improvement, observational study in a single primary care office.

\section{Data collection}

Blood pressures were measured in a single PCP office (Chelsea Family and Internal Medicine, IHA, Chelsea, MI) by medical assistants (MA) for all patients that were seen for an office visit, as is the usual custom. The patient was instructed to sit in a chair with their back supported and feet on the ground without talking nor arm movement. The MA used an electronic timer on their computer. The patient was interviewed by the MA and the timer was started afterwards and immediately prior to the medical assistant standing to take the patient's BP. The timer was stopped immediately after the medical assistant sat down and before entering the BP into the electronic medical record. The total time was recorded on separate sheet along with the patient's name, birthdate, measured BP, BMI (Body Mass Index) and gender. The paper data was transferred by the author into an Excel spreadsheet.

Blood pressure measurements were performed using an oscillometric Welch Allyn Connex Vital Signs Monitor (Welch Allyn Connex Vital Signs Monitor, Skaneateles Falls, NY, USA) until at least 400 readings were obtained. Manual auscultatory readings with a wall mounted Tycos device (Tycos, Skaneateles Falls, NY, USA) were subsequently used by the MA until another minimum of 400 readings were measured. The BP measurements were performed over a 43-day period. BP measurements were taken during office visits by two physicians and their assigned MA measured the readings. Approximately six MA took the measurements and the MA and physicians were not blinded to the nature of the quality improvement project. The aggregate data was blinded to all the MA.

The primary outcome was the time to measure BP by each method. The secondary outcomes were the difference in systolic and diastolic blood pressures for each method, BP time/age, BP time/BMI, BP time/readings over the measured month, and the estimated cost per BP measurement.

This study was a quality improvement project by the practice per the Saint Joseph Mercy Health System Ann Arbor, MI-Institutional Review Board and a formal IRB approval was not needed.

\section{Statistical analysis}

Microsoft Excel statistical functions were used to calculate for standard deviation, student t-test (2-tail, type-2), and averages. As described in the data collection section, 
oscillometric measurements were taken consecutively for the first set of patient visits followed by manual collections on the next set of patients.

Descriptive statistical summaries were computed for the differences between the two methods grouped into $1^{\text {st }}$ third, $2^{\text {nd }}$ third and $3^{\text {rd }}$ thirds of the measurements taken with each method. In particular, mean, standard deviation, and the five number summaries (Minimum, 25th percentile, Median, 75th Percentile, Maximum) of the differences were assessed. $95 \%$ confidence intervals for the mean differences were computed.

Bland-Altman plots were generated to display the agreements between the measurements for readings that were grouped into $1^{\text {st }}$ third, $2^{\text {nd }}$ third and $3^{\text {rd }}$ thirds of the measurements taken with each method. Scatter plots were used to show trends for Time taken per measurement vs. systolic, diastolic, BMI and order of measurements. Age was computed using the date of data collection and the birthdate i.e. age $=$ (data collection date-birthdate)/365. Significant differences between groups were reported at the $\alpha$-level of 0.05 .

Additional output/data analysis for this paper was generated using SAS software (Version 9.4 of the SAS System for Windows. Copyright (c) 2002-2012 SAS Institute Inc. SAS and all other SAS Institute Inc. product or service names are registered trademarks or trademarks of SAS Institute Inc., Cary, NC, USA), which replicated the Excel calculations.

\section{Results}

A total of 827 subjects had their BP measured during a routine office visit. The Welch Allyn Connex Vital Signs Monitor (WA) was used for the first 411 subjects and manual auscultation (Manual) was used for the next 416 subjects (Table 1 ). The average age (64.1 \pm 16.6 years old-WA, $64.8 \pm 16.9$ years old- Manual) was similar in both groups $(\mathrm{p}<0.05)$, however there was a higher male average $(68 \%)$ in the WA group compared to the Manual (61\%) group. There was a slight trend toward higher age of the subjects in both groups which resulted in slightly longer measurement times (Figure 1). The Manual group also had a slightly higher BMI (30.9 \pm 7.2$)$ compared to the WA group (29.7 \pm 6.5$)$, $\mathrm{p}=0.01$ ). Smaller BMI subjects had quicker measurement times for the WA group compared to the Manual group (Figure 1), however this equalized at the higher BMI.

The average time (Table 1 ) to manually measure BP (Manual) was 58.6 \pm 13.9 seconds (range 15-146 seconds, , whereas the WA average was $39.8 \pm 23.0$ seconds (range 14-124 seconds, \pm 23.0 ), 18.8 seconds faster $(\mathrm{p}<0.05)$. The MA demonstrated a learning effect with the WA as the measurements became quicker as they emphasized to the patients not to move their arms during the measurements. This is seen in figure 2 . The average time for BP measurement was not significantly different between Manual

\begin{tabular}{|c|c|c|c|c|}
\hline Table 1: Demographics and results. & WA & Manual & \\
\hline N & 411 & 416 & & \\
\hline Male\% & $68 \%$ & $61 \%$ & & \\
\hline Female\% & $32 \%$ & $39 \%$ & & \\
\hline Age & 64.1 & 64.8 & & P \\
\hline BMI & 29.7 & 30.9 & & $<0.05$ \\
\hline & WA & Manual & Defference & 0.16 \\
\hline Times (Seconds) & 39.8 & 58.6 & 18.8 & $<0.05$ \\
\hline Times (Seconds) First 1/3 readings & 56.1 & 59.1 & 3 & $<0.05$ \\
\hline Times (Seconds) Last 1/3 readings & 29.3 & 57.5 & 28.3 & $<0.05$ \\
\hline SBP (mmHg) & 136.3 & 126.4 & -9.9 & -5.9 \\
\hline DBP (mmHg) & 8.2 & 74.3 & $\$ 0.11$ & \\
\hline Cost/avg BP & $\$ 0.24$ & $\$ 0.35$ & $\$ 0.02$ & \\
\hline Cost/avg BP (First 1/3) & $\$ 0.33$ & $\$ 0.35$ & $\$ 0.17$ & \\
\hline Cost/avg BP Last 1/3 & $\$ 0.17$ & $\$ 0.34$ & & \\
\hline
\end{tabular}




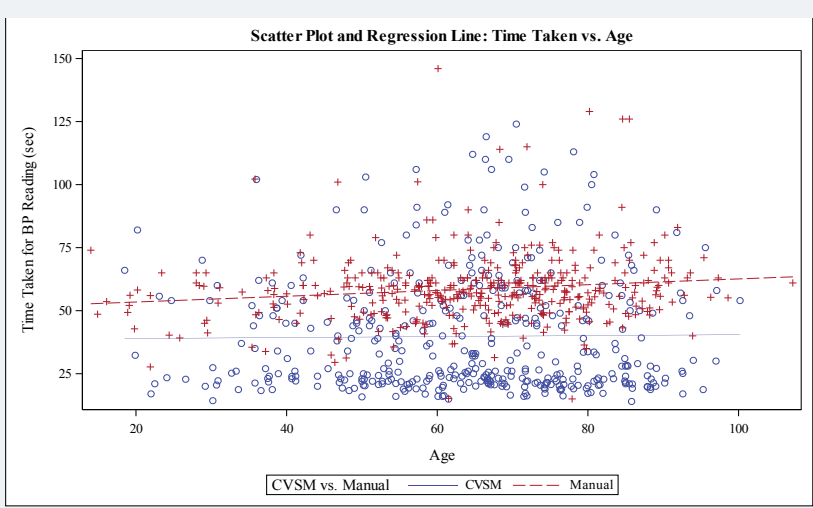

Scatter graph of Age of subjects (years) vs. Time of measurement (seconds)

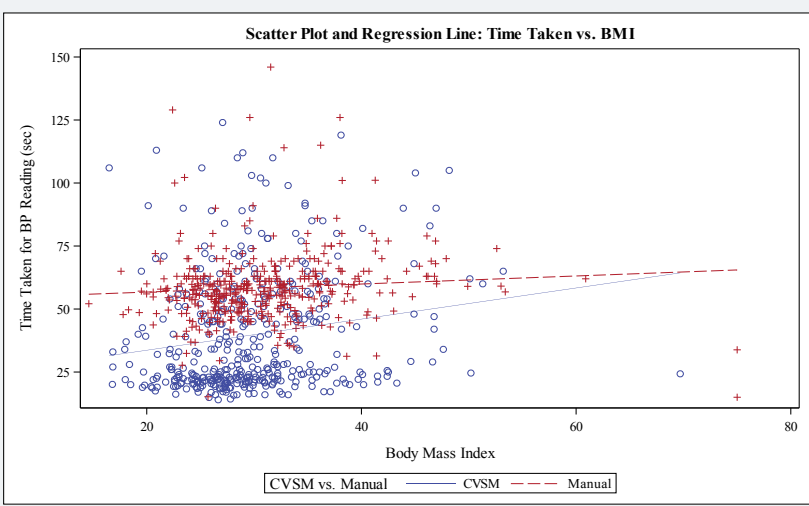

Figure 1: Scatter graph of Age of subjects (years) vs. Time of measurement (seconds); Scatter graph of BMI (kg/ $\mathrm{m}^{2}$ ) vs. Time of measurement (seconds).

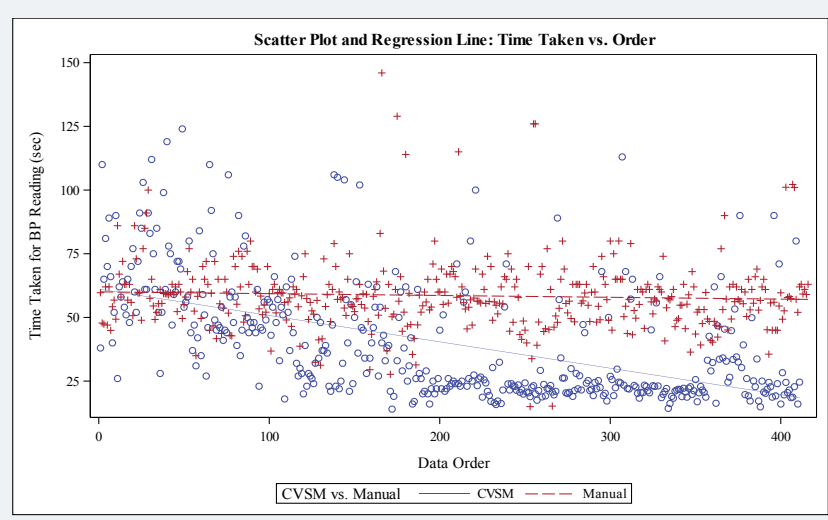

Scatter graph of Time of measurement (seconds) vs. order of readings

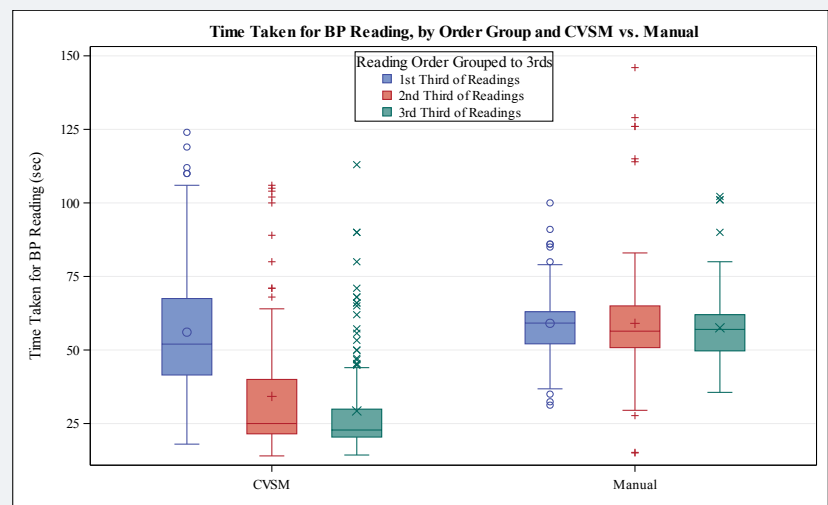

Bland Altman Plot of Time of measurement (seconds) vs. Order of measurements

Figure 2: Scatter graph of Time of measurement (seconds) vs. order of readings; Bland Altman Plot of Time of measurement (seconds) vs. Order of measurements. 
(59.1 seconds) and WA (56.1 seconds) for the first $1 / 3$ of measurements ( $p=0.16$ ). The last $1 / 3$ of measurements showed significant improvement $(p<0.05)$ in the speed of measurement for WA (29.3 seconds) vs. Manual ( 57.5 seconds), but the Manual did not significantly change between the first $1 / 3$ and last $1 / 3$ of measurements $(p=0.25)$.

The average BP for WA was 136.3/80.2 mmHg, which was higher than the Manual average of 126.4/74.3 mmHg. The WA and Manual groups measured different patients and the study was not designed to determine the accuracy of either measurement technique. The groups were randomly matched for age and approximately matched for BMI, however the WA group had a slightly higher proportion of males/females than the Manual group. The time to measure diastolic BP when it was higher compared to lower was less for both groups (Figure 3). The time to measure systolic BP was when it was higher compared to lower was about equal for WA and slightly less time for the Manual method (Figure 3).

Our MA cost was based on salary plus benefits of $\$ 21.37$ per hour or $\$ 0.01$ per second. The average MA cost to measure a single BP using the Manual method was $\$ 0.35$ vs. the WA method was $\$ 0.24$, or a saving with the WA method of $\$ 0.11$ per measurement. After the proper technique was mastered, the cost of the last $1 / 3$ of measurements for the Manual was virtually the same at $\$ 0.34$, however the WA method decreased to $\$ 0.17$, a saving of $\$ 0.17$ per measurement.

The Manual method had a trend for longer measurement times in the elderly vs. younger subjects, however the WA method did not show this trend. Smaller BMI had markedly quicker measurements with the WA method and this was similar but slightly less for Manual readings. The larger cuff size (longer inflation time) needed for larger arm was the likely explanation for this. Higher systolic BP measurements were slightly quicker with the Manual method and minimally different with the WA method. Higher diastolic BP measurements were also quicker for the Manual and WA methods.

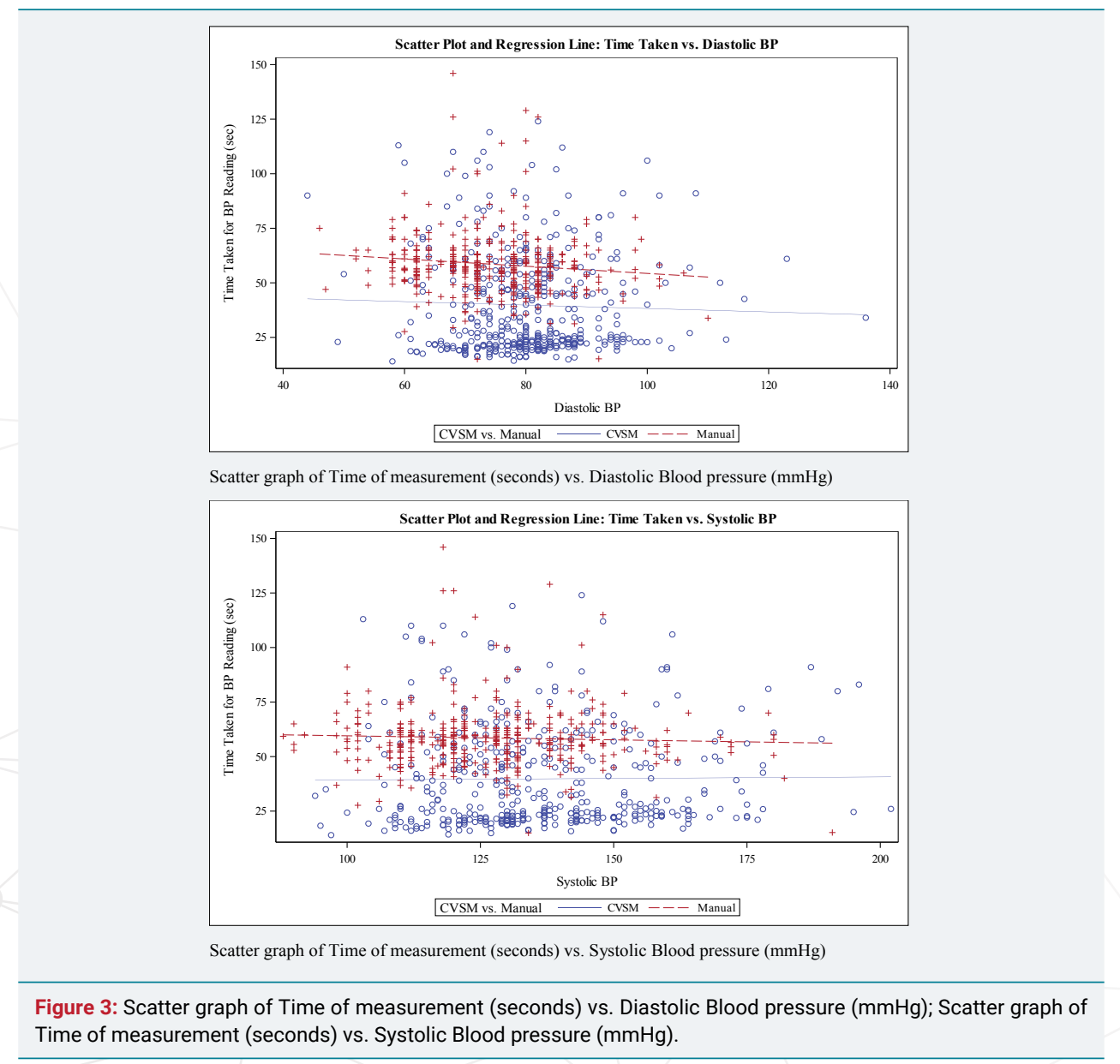


Sixty-nine patients had second visits during the study period. Five patients had manual readings during both visits and the time difference between each patient's reading was $1.6( \pm 1.8)$ seconds. Fifty patients had a reading first by the WA method and at a subsequent visit by the Manual method. The time difference for this group was $17.2( \pm 29.2)$ seconds. Fourteen patients had the WA method at both visits with a 14.3 $( \pm 40.0)$ second difference.

\section{Limitations}

This was a non-blinded single center observational study. The MA performed the time study and understood that the study was to determine the potential timesaving of using the WA device, which they desired to use. None of the MA reviewed the composite data prior to the end of the study. The costs of MA salaries can vary based on different regions of the country as well as size of the practice.

\section{Discussion}

Measuring BP correctly is a tedious, time consuming vital sign. I state this based on my experience of almost 36 years of performing over 34,000 measurements equating to 33,726 minutes of BP measurements (562 hours). The manual blood pressure measurement (Manual) in this study averaged 58.6 seconds. The Welch Allyn Connex Vital Signs Monitor WA is capable of measuring systolic BP on inflation by using SureBP ${ }^{\circledR}$ technology, allowing quicker measurements than other traditional oscillometric devices which commonly measure systolic BP on deflation. The automatic WA method averaged 39.8 seconds however with device experience this decreased to 29.3 seconds (Table 1, Figures 3,4). The Manual method did not change with experience. The cost savings using the WA automatic method after experience with this device equated to 17 cents per measurement compared to manual auscultation. Although this does not appear to be a substantial savings, BP is measured per Primary Care Physician for every patient visit. If you assume 25.6 patient visits per day, 5 days per week for 52 weeks, as was performed in this study, this savings is $\$ 1,119$ per year.
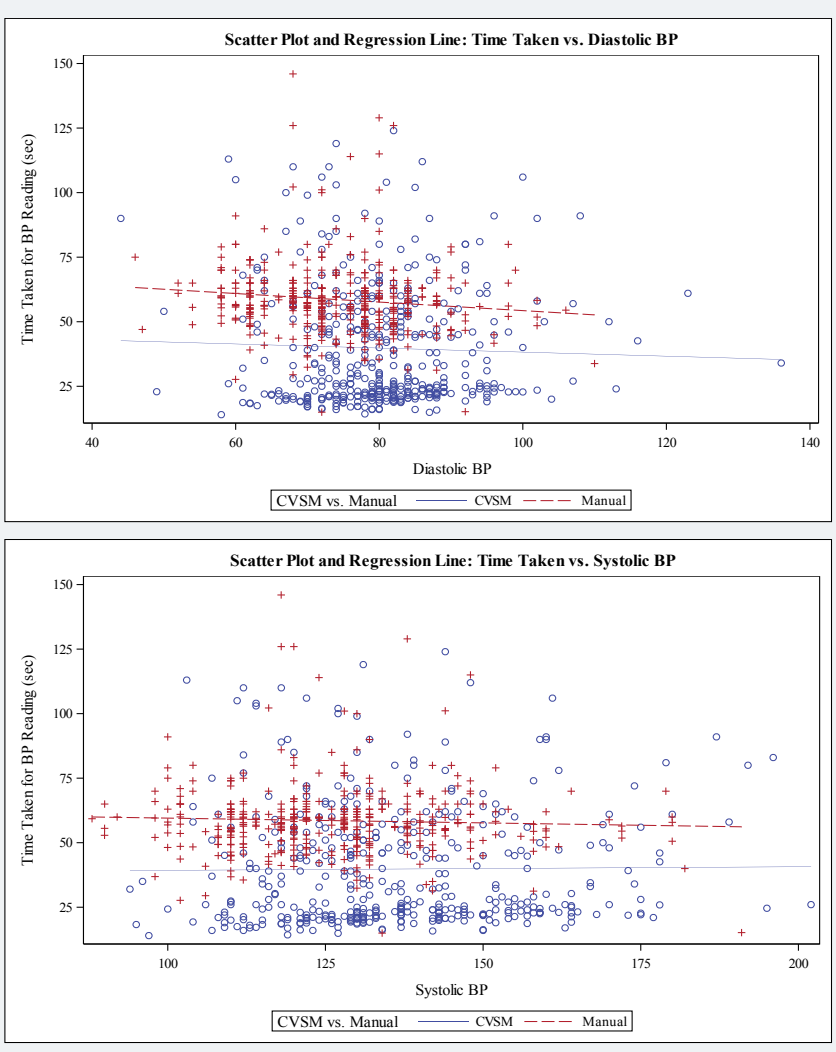

Figure 4: Scatter Plot and Regression Line: Time Taken vs. Diastolic BP; Scatter Plot and Regression Line: Time Taken vs. Systolic BP. 
The WA method measured the BP higher $(136.3 / 80.2 \mathrm{mmHg})$ than the Manual method (126.4/74.3 $\mathrm{mmHg})$ by $9.9 / 5.9 \mathrm{mmHg}$. While both methods had similar demographics, the study was not designed to match BP readings in both groups. The Manual auscultatory method should inflate the cuff $30 \mathrm{mmHg}$ above the point at which the radial pressure disappears and the deflation rate should be $2 \mathrm{mmHg}$ per second [4]. I am not aware of readings performed in any clinical practice whereby the peak auscultatory cuff inflation is measured by $30 \mathrm{mmHg}$ above the point the radial pressure disappears. Another explanation as to why Manual is lower than WA is either the error of not inflating the manual cuff enough (artificially lowering the systolic BP) or too rapid deflation (artificially lowering systolic and diastolic BP). Clinically, most inflations arbitrarily occur at approximately $180 \mathrm{mmHg}$. In the Manual method in this study, the average time to deflate our average BP of $126.4 / 74.3 \mathrm{mmHg}$ at a deflation of $2 \mathrm{mmHg}$ the measurement time should be 52.8 seconds. The actual average study measurement was at approximately this rate ( 58.6 seconds). It was faster to measure patients with higher compared to lower diastolic BP for both groups likely due to lower pulse pressures (Figure 3).

Measuring BP fast and efficiently do not equate to accuracy, compared to research BP techniques or daytime ambulatory monitoring. Proper BP measurement requires time and skill, both of which are sometimes lacking for this redundant task in nonhypertension specialty offices for these hurried office personnel. Expecting the patients to sit down for 5 minutes prior to BP measurement is often unrealistic as the office visit may only be scheduled for 10-20 minutes. In our office, the MA rooming time prior to the physician interaction is between 5 to 10 minutes per patient. The auscultatory measurement of 58.6 seconds is thus $10-20 \%$ of the MA time to room the patient. The WA method would save $6-12 \%$ of this rooming time and this does not account for the Welch Allyn Connex Vital Signs Monitor capability of multiple readings and Bluetooth connectivity to directly add the vital signs into the electronic record. This function would assure accuracy and likely saving another 5 seconds. Additional optional functions of this monitor include finger oximetry and body temperature measurement which would also be Bluetooth connected and would further improve the MA rooming time.

A proposal to improve Primary Care office BP accuracy by using non-observer patient-resting measurements [2,3]. This method could decrease the rooming MA time, however utilizes an often-limited examination room resource for 5 to 6 minutes. The fixed cost of an examination room is variable, however likely $\$ 0.02-0.05$ per minute of usage, however if there are limited examination rooms the lost opportunity of physician billing could cost $\$ 35-41$ for 7 to 8 minutes of room usage. This is substantial for a primary care office.

\section{Conclusion}

The WA method saved 18.8 seconds compared to the Manual method. The potential return on the investment could be appropriately one year. Perhaps it is time to standardize BP measurement and stop measuring BP in primary care offices by the auscultatory method.

\section{Implications}

Most research has transitioned from measuring BP by auscultation to the oscillometric method over the past decade. This was also a silent transition likely to avoid the bias error inherent in the auscultatory method including rapid deflation, end-zero bias, and visual and hearing impairments. The WA oscillometric method showed time/cost efficiency in our practice. Perhaps it is time to standardize BP measurement and stop measuring BP in primary care offices by the auscultatory method. Hypertension treatment could be most improved by more reliance on proper home BP or 24-hour ambulatory monitoring. 


\section{Author's contributions}

SAY helped design the study, conduct the study, analyze and interpret the data, and draft and critically revise the manuscript. SAY is the guarantor of the content of the manuscript, including the data and analysis. SAY read and approved the final version of the manuscript.

\section{Supported by: Welch Allyn}

Role of the Sponsor: Welch Allyn provided the Connex Vital Signs Monitor without charge and the data was replicated with their statistician. They had no input into the design, analysis, writing, nor review of the manuscript.

\section{References}

1. Centers for Disease Control and Prevention. Ambulatory Care Use and Physician office visits. 2017; Ref.: https://goo.gl/3JdzAT

2. Myers MG, Godwin M, Dawes M, Kiss A, Tobe SW, Kaczorowski J. Measurement of Blood Pressure in the Office: Recognizing the Problem and Proposing the Solution. Hypertension. 2010; 55: 195-200. Ref.: https://goo.gl/3JdzAT

3. SPRINT Research Group, Wright JT Jr, Williamson JD, Whelton PK, Snyder JK, et al. A Randomized Trial of Intensive versus Standard Blood-Pressure Control. N Engl J Med. 2015; 373: 2103-2116. Ref.: https://goo.gl/577UR4

4. Pickering TG, Hall JE, Appel LJ, Falkner BE, Graves J, et al. Recommendations for Blood Pressure Measurement in Humans and Experimental Animals, Part 1: Blood Pressure Measurement in Humans: A Statement for Professionals from the Subcommittee of Professional and Public Education of the American Heart Association Council on High Blood Pressure Research. Circulation. 2005; 111: 697716. Ref.: https://goo.gl/o81j3Y 